\title{
High Precision CAE Analysis of Automotive Transaxle Oil Seal Leakage
}

\author{
Yasuaki Nozawa, Takahiro Ito, Kakuro Amasaka \\ Aoyama Gakuin University, Kanagawa-ken, Japan
}

\begin{abstract}
The manufacturing industry today due to worldwide competition is focused on shorter development cycle. In this situation, computer aided education (CAE) technology as a tool for simultaneous achievement of quality, cost and delivery (QCD) plays an important role. The hardware-software environment surrounding CAE has evolved. Though technological problems have been understood and general solutions have been derived and reflected in the CAE analysis software, research findings that boost the credibility of CAE have still not been incorporated fully enough into the development of design process. The real technical mechanism issue is not precisely capture. Therefore, it is important to clarify the real cause in CAE results through CAE simulation in order to assure product reliability and assurance. It is the aim of this study to realize the prediction of design analysis process through understanding of unclear technical mechanism in abnormal occurrences with the utilization of CAE simulation. In other words, it is the aim of this study to focus on issue in automotive transaxle oil seal leakage to understand, grasp, and visualize the main cause through usage of CAE analysis process. It is understood that the point of contact and pump volume was related and this could contribute towards seals quality design. Plus, the utilization of CAE analysis in prediction phase to realized design development is also possible.
\end{abstract}

Keywords: oil seal, optimized design using high-quality assurance CAE, digital engineering

\section{Introduction}

The manufacturing industry today due to worldwide competition is focused on shorter development cycle. In this situation, computer aided education (CAE) technology as a tool for simultaneous achievement of quality, cost and delivery (QCD) plays an important role. The hardware-software environment surrounding CAE has evolved. Though technological problems have been understood and general solutions have been derived and reflected in the CAE analysis software, research findings that boost the credibility of CAE have still not been incorporated fully enough into the development of design process. The real technical mechanism issue is not precisely capture. Therefore, it is important to clarify the real cause in CAE results through numerical simulation (CAE analysis) in order to assure product reliability and assurance (Amasaka, 2008).

Concretely, authors take up the automotive transaxle oil seal leakage. As the result, the difference was seen by the material and the shape of the oil seal. In addition, CAE analysis was carried out to catch behavior of

Yasuaki Nozawa, Graduate Student, School of Science and Engineering, Aoyama Gakuin University.

Takahiro Ito, Graduate Student, School of Human Sciences and Engineering, Aoyama Gakuin University.

Kakuro Amasaka, Ph.D., Professor, School of Science and Engineering, Aoyama Gakuin University.

Correspondence concerning this article should be addressed to Kakuro Amasaka, 5-10-1, Fuchinobe, Chuo-ku, Sagamihara-shi, Kanagawa-ken, 252-5258, Japan. E-mail: kakuro_amasaka@ise.aoyama.ac.jp. 
oil and the amount of the pump on the surface of the seal of three types divided depending on two parameters that showed the characteristic of the seal based on the result of the real machine experiment. It achieved the desired results (Amasaka, 2007, 2010).

\section{About CAE Current Status and Issues}

CAE and other numerical simulations have been applied to a wide variety of business processes in recent years, including research and development, design, preproduction and testing/evaluations, production technology, production preparation, and manufacturing. Despite these high expectations, conventional forms of CAE analysis resulted in figures that deviated as much as $10 \%-20 \%$ from those obtained through prototype testing evaluations.

This means that many companies are now stuck with applying CAE only to the monitoring task of comparative evaluations of old and new products—-despite the enormous amount of funds they have invested in CAE development. There are two absolute requirements for precise (highly-reliable) CAE analysis methods that can both prevent the critical technical problems plaguing manufacturers from recurring and contribute to new product designs. The first is reducing the deviation from prototype testing evaluation figures to $5 \%$ or less, and the second is evaluating the absolute values needed for tolerance designs

Figure 1 shows a highly-reliable CAE analysis technology component model that should be installed in any piece of CAE analysis software that seeks to generate highly-reliable CAE analysis results. A detailed explanation of this figure follows. During the implementation phase of CAE analysis, the user first defines the problem to be solved (1). Next, the system begins working on the problem by, for example, generating several mathematical formulas (2). During the CAE analysis, a computer is used as a tool for analyzing the model (3).

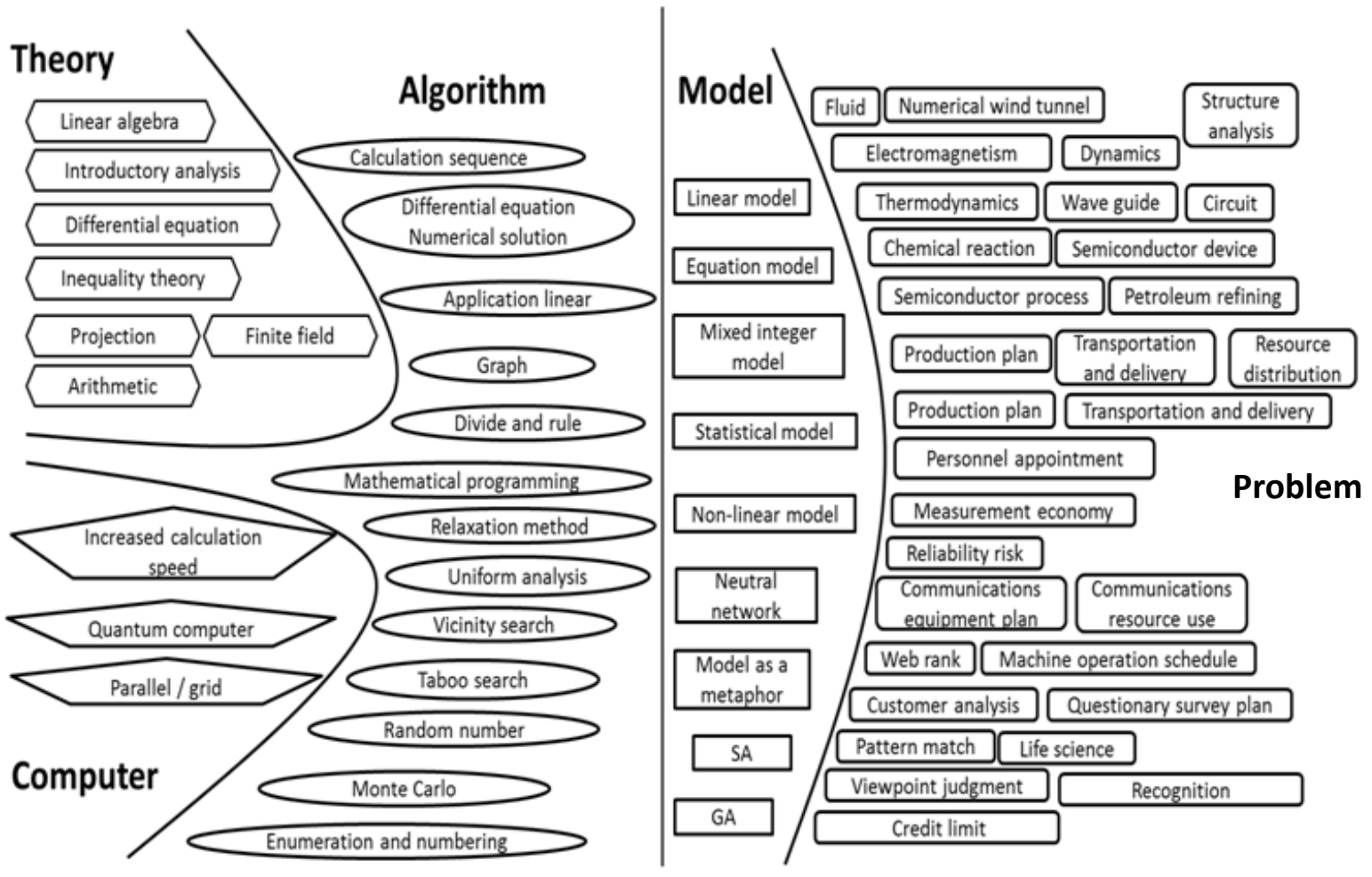

Figure 1. Highly-reliable CAE analysis technology element model. 
Note that the analysis tool must be a calculation tool (software) capable of performing the operation; in other words, it must be given as an algorithm. Here, the calculation results must be driven by some kind of theory indicating the suitability, applicable scope, performance, and expected precision of the algorithm itself (4). Obviously, the success of the CAE analysis is a major factor in determining the technological competence of the computer performing the calculations, as it is what allows the algorithm to be put into action (5). The success of the CAE analysis affects the overall strength of technology components (Amasaka, 2007; Magoshi, Fujisawa, \& Sugiura, 2003).

Hardware-software environment that surrounds CAE has evolved: "Technological problem” is reflected, "general solution" is derived, however, the "finding of the problem-solving" is reflected in the CAE analysis software, and the research case where the credibility of CAE is improved is not established for the development design process still enough. As a result, the prediction result of CAE is not reproduced by the experiment and the re-doing of CAE and the experiment is generated, it is a current state that the reduction in the faster time to market and the development cost is not advanced except the desire.

Using these causal factors as an example, the following issues can be identified through a comparison of the relationship diagrams of Figure 1.

(1) Analytical scope issues (problem): With a large analytical scope, strategies such as partially simplifying form are attempted as a way of reducing that scope. If these strategies are inappropriate, it may affect the reproducibility of the experiment;

(2) Modeling issues (model): When an analysis targets a phenomenon that has not been adequately explained in terms of theory, the numerical analysis model may end up diverging from the way the phenomenon is actually manifesting;

(3) Algorithm issues (algorithm): If appropriate modeling is not carried out based on an accurate understanding of the applicable scope and particular characteristics of a given algorithm, or if an algorithm is not properly chosen or effectively implemented, the resulting analysis will not be sufficient;

(4) Issues with input criteria (theory): If the physical properties of a component are difficult to measure, for example, it may lead to an inaccurate grasp of physical features such as temperature dependence. If the input criteria for material properties do not match the actual item exactly, the discrepancy will compromise the precision of the analysis;

(5) Analysis issues (computer technology): The precision of the computer technology itself, unrealistic calculations, or a lack of skill on the part of the analyst can make it impossible to carry out a proper analysis or can generate significant gaps.

\section{High Precision CAE Analysis of Automotive Transaxle Oil Seal Leakage}

It is the aim of this study to realize the prediction of design analysis process through understanding of unclear technical mechanism in abnormal occurrences with the utilization of CAE simulation. Specifically, a CAE analysis was implemented on the oil used to lubricate the space between the oil seal and the axis based on the insights gained from visualizing this technology.

\section{Visualization of the Automotive Transaxle Oil Seal Leakage Problem}

Automotive transaxle oil seal leakage problem. The problem of leaking oil seals is a technical issue where uncertainties still remain in terms of the technological mechanism. Oil leaks result in insufficient lubricant, which leads to excessive friction between the oil seal and axis. This in turn generates frictional heat, 
which causes the seal component (which is made of synthetic rubber) to burn into the axis, hindering rotational movement. Because of the oil seals used in drivetrains are in the drive shaft (which directly affects the driving performance of the vehicle), there is a danger that any malfunction might potentially result in a lethal accident.

In addition, As oil seals are used within the mechanical portions of a vehicle, repairs are expensive. Table 1 lists leak volume guidelines based on oil leak frequency in areas containing Idemitsu Kosan lubricant. The table indicates that even a small oil leak is impossible to ignore, as even one producing a single drop every 10 seconds can result in the loss of 0.42 liters per day or 12.6 liters per month. These facts make it clear that highly-reliable oil seals are a critical mechanical element in automobiles.

Table 1

Leak Volume Guidelines Based on Oil Leak Frequency $(L)$

\begin{tabular}{lccc}
\hline Leak volume guidelines (L) & /one day & /one month & /one year \\
\hline One drop every 10 seconds & 0.42 & 12.6 & 151 \\
One drop every five seconds & 0.85 & 25.5 & 306 \\
One drop every one second & 4.25 & 127.5 & 1,530 \\
Fall into filament form (small) & 24 & 720 & 8,640 \\
Fall into filament form (medium) & 91 & 2,730 & 32,760 \\
Fall into filament form (large) & 176 & 5,280 & 63,360 \\
\hline
\end{tabular}

Problem identification. First, in order to clarify what information is needed for the visualization experiment, it is essential to understand what factors cause oil leak problems, sorting these into both known and unknown factors. The study of the oil leak mechanism involved looking at different issues affecting the leak through several studies, as indicated in Figure 2.

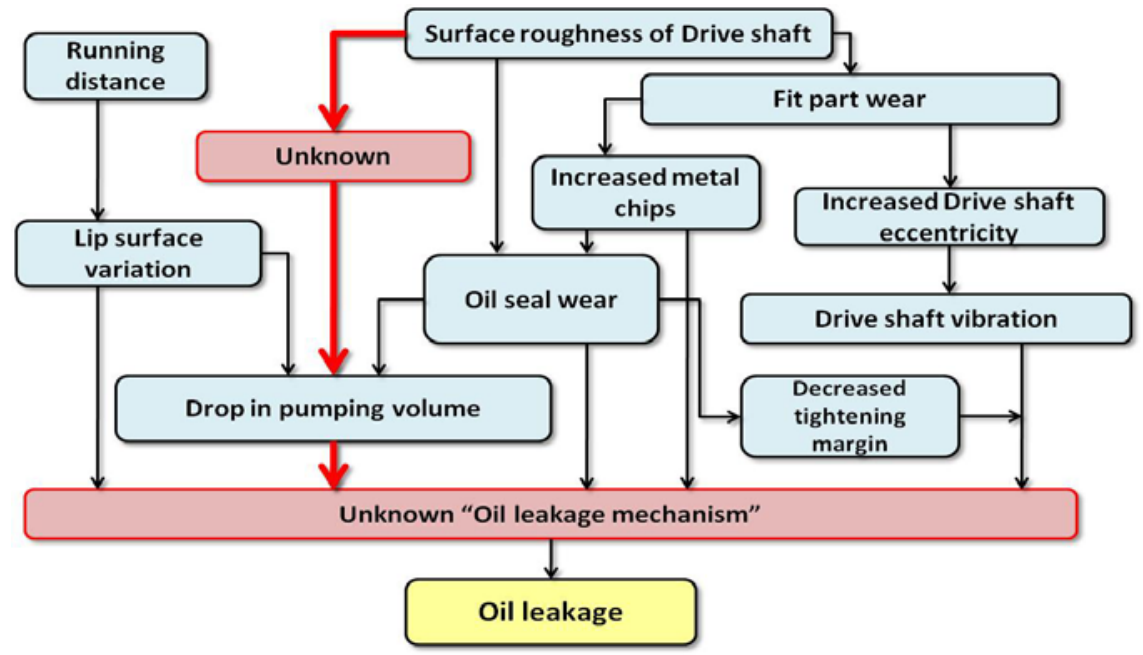

Figure 2. Estimation of the oil leakage mechanism.

The factor and the knowledge that related by using the relation drawing to search for the causal relation of the oil leakage that had been obtained so far were arranged. Because the oil leakage mechanism is uncertain, the generation route is not clarified, and the part where making the dynamic behavior visible is necessary is specified, and it experiments with the device of making to visible (Amasaka, 2007).

Visualization of dynamic behavior by visualization experiment. In order to visualize the dynamic behavior of the oil seal lip, the visible making the oil seal device shown in Figure 3 was developed. The oil seal 
was reproduced as shown in figure, and it soaked in the lubricant in a similar way, it changed into the glass axis that rotated the drive shaft eccentrically by way of the spindle motor, and operation in an actual vehicle was reproduced with the transaxle.

The effect of sealing up of the oil seal lip used and visualized the optical fiber. The experiment of the observation on the sliding side did with a seal of three types with different material and shape, and caught the state on sliding surfaces and the relations of the leakage stopping (Amasaka, 2007; Amasaka \& Osaki, 2003; Steinberg, 1996; Takahashi, Ueno, Yamaji, \& Amasaka, 2010).
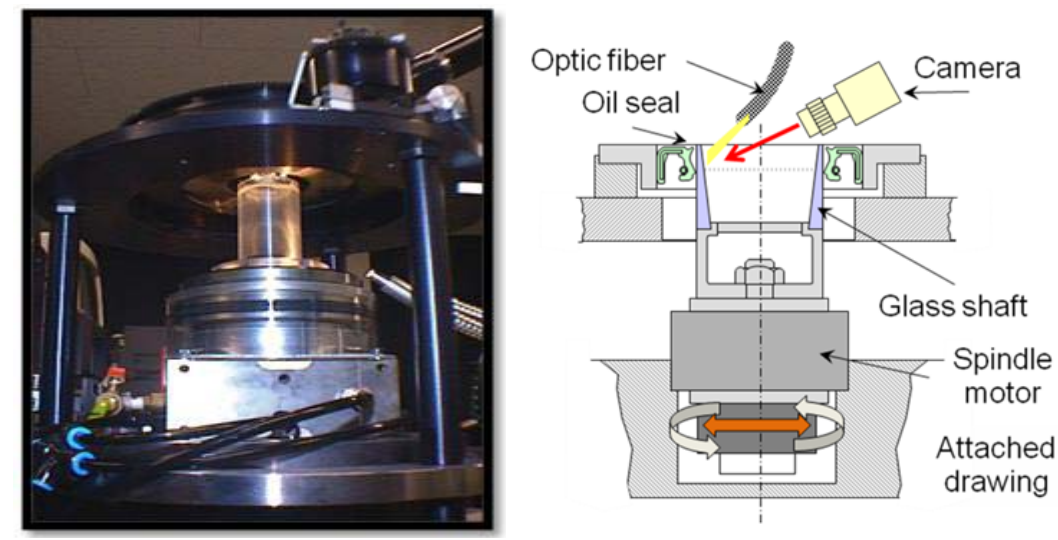

Figure 3. Outline of device by visualization.

The non-lubrication criteria (conditions indicating a lack of oil) in Figure 4 show a structural form diagram of the sliding surface. Specifically, two characteristic values are indicated as a way of quantifying the form of the sliding surface.

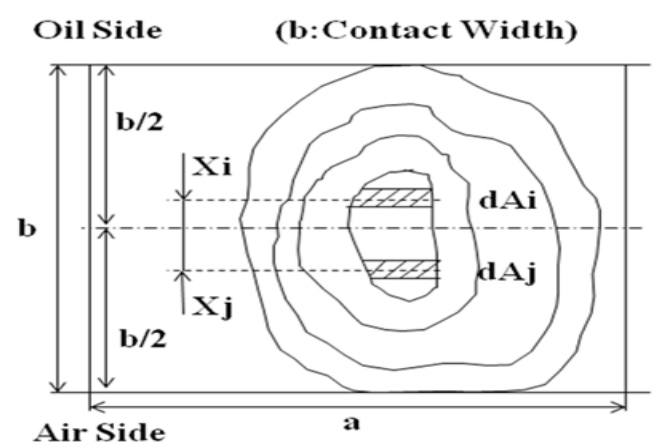

Figure 4. Pattern diagram of the sliding side.

$$
\begin{gathered}
A_{R}=\frac{\text { The real contact area }}{\text { The visible touch area }}=\frac{(\text { Shaded area })}{(a \times b)} \\
X_{G}=\frac{\sum d A i\left(\frac{X i}{b / 2}\right)-\sum d A j\left(\frac{X j}{b / 2}\right)}{\sum d A i+\sum d A j}
\end{gathered}
$$

The first is the ratio $A_{R}$, which indicates the proportion of the visible touch area that is the true contact area (Formula (1)). The shaded area shows the real contract area, defining a characteristic value that indicates the degree of roughness on the sliding surface. A small $A_{R}$ ratio indicates minute rough spots on the sliding surface, 
while a large $A_{R}$ value indicates seal exhibiting uniform contact. The second characteristic value is $X_{G}$, which indicates the degree to which the true contact area leans towards the oil side (Formula (2)). A positive $X_{G}$ value indicates that the true contact area is leaning towards the oil side, while a negative value indicates that it is leaning towards the atmospheric side.

Using these two characteristic values, authors are able to quantify three types of oil seals which are summarized in Table 2. Of the three, only Seal 1 achieves a hermetic seal; oil leaks were observed in the other two. An examination of the relationship between the two characteristic values and leak stoppage was further conducted for a variety of different seal types. These results are shown in Figure 5. This leak stoppage map indicates that the characteristic values can be used to express seal criteria. In other words, the study yielded the insight that a characteristic sliding surface structure that maintains a sealed state requires minute surface roughness (the presence of minute protrusions) and a true contact area that leans towards the oil side.

Table 2

Outcome of the Experiments

\begin{tabular}{lllc}
\hline & Type 1 & Type 2 & Type 3 \\
\hline$X_{G}$ & 0.02 & 0.47 & 0.04 \\
$A_{R}$ & 0.14 & 0.05 & -0.21 \\
Result & Sealing & Leakage & Leakage \\
\hline
\end{tabular}

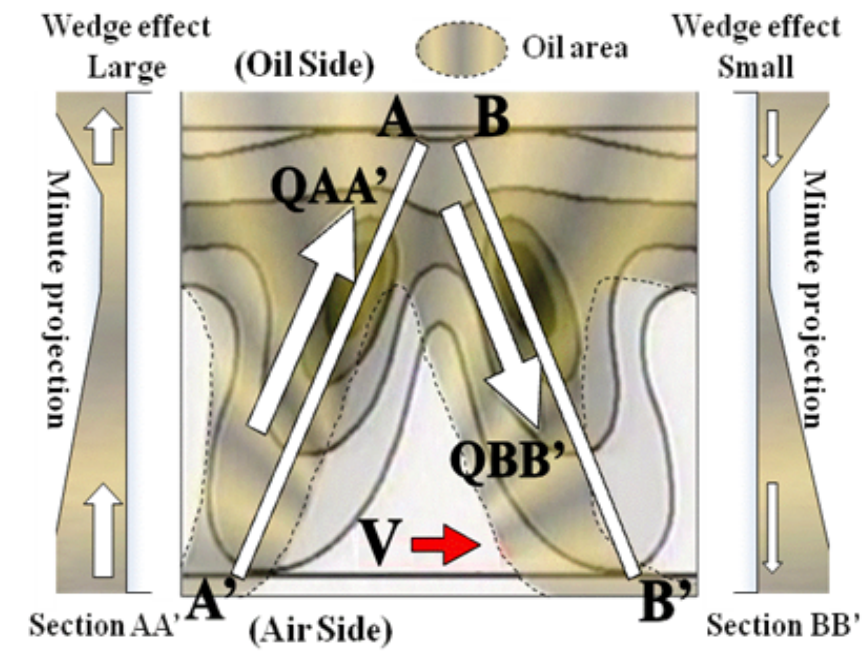

Figure 5. Oil seal leak stoppage map using characteristic values $A_{R}$ and $X_{G}$ for sliding surface form.

Logical reasoning. The relationship between oil seal surface conditions and oil leak stoppage that was revealed in the visualization experiment analyzed (1) pump volume; and (2) the behavior of oil between the seal and axis while considering parameters that described the condition of the seal surface. A basic structural model of the sliding surface that dictates seal conditions is shown in Figure 6. The model shows the minute roughness that exists on the sliding surfaces by statistically approximating a central projection.

Sealing of the sliding surface is achieved when QAA' > QBB', where QAA' is the volume of oil flowing into section $\mathrm{AA}^{\prime}$ and $\mathrm{QBB}$ ' is the volume of oil flowing out of section BB'. In addition, if the pump volume is taken to be the difference between QAA' and QBB', authors can express the fact that proper pump volume is being maintained with QAA'> QBB', and low pump volume with QAA'< QBB'. Authors can now proceed to 
an analysis of this basic structural model during the CAE analysis implementation phase (Ito, Sato, Yamaji, \& Amasaka, 2010; Nakamura, 1987; Amasaka, Yamaji, \& Ito, 2010; Nakamura, Kameike, \& Ono, 2000; Toda, Nakamura, Ono, \& Sato, 1999).

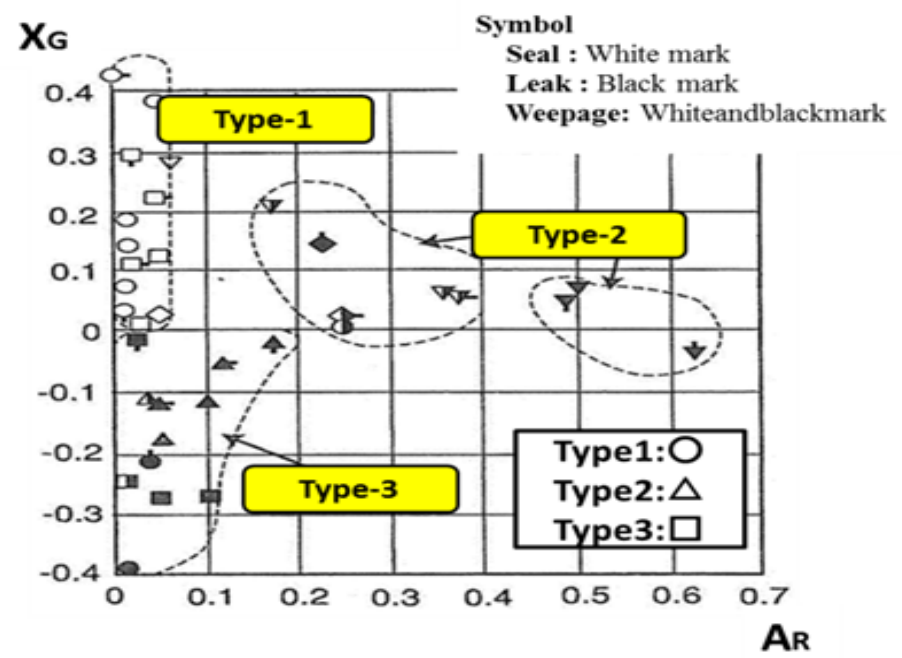

Figure 6. Sliding surface basic structure model.

Technological elements of the oil seal simulator. Figure 7 shows the technological elements that the oil seal simulator should have. In handling the oil leakage phenomenon, the problem is grasping the pump volume and lip side pressure distribution that directs the behavior and the circulation pattern of the oil on the minute projection area of sliding surfaces.

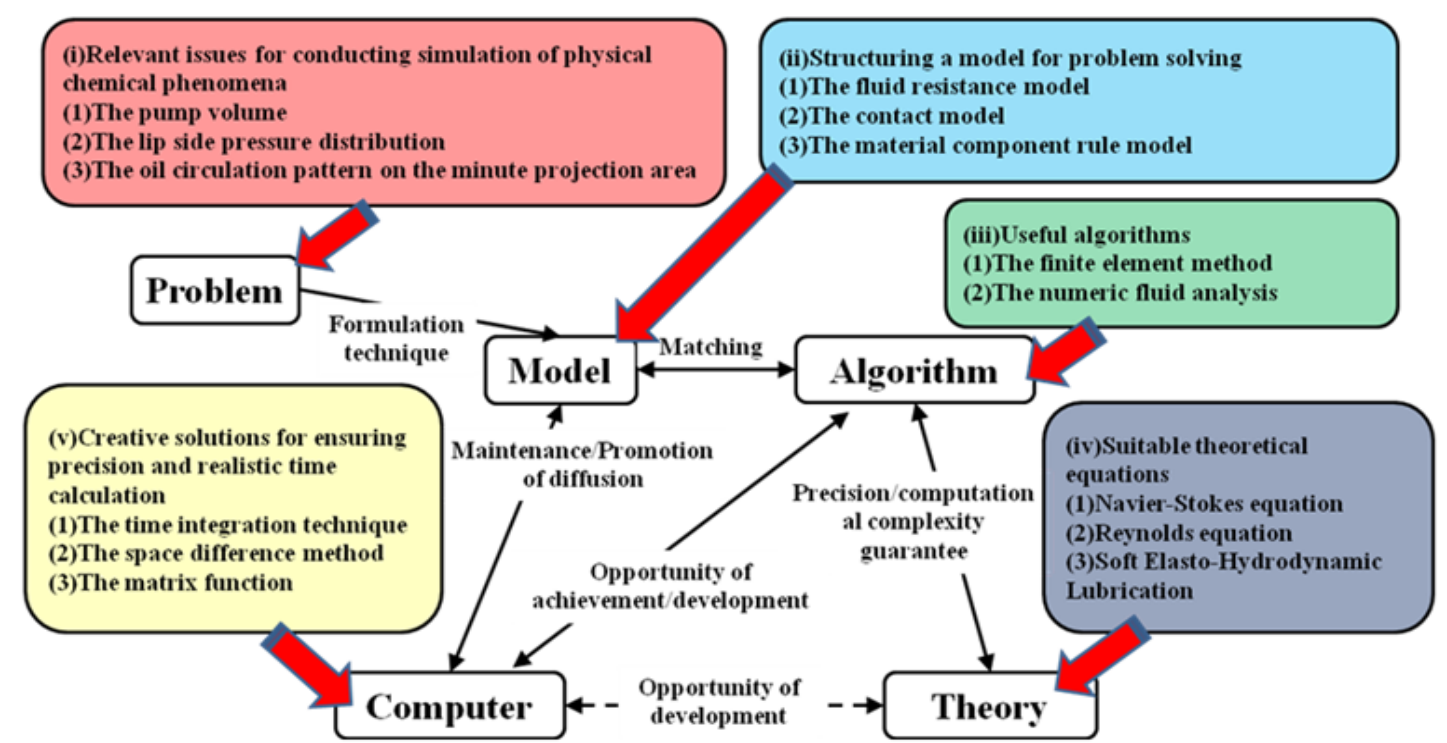

Figure 7. Technological elements of the oil seal simulator.

The fluid resistance model, the contact model, and the material component rule model are used to solve these problems. The finite element method and numeric fluid are analyzed as a convenient algorithm. The Reynolds equation, Soft Elasto-Hydrodynamic Lubrication, and Navier-Stokes equation are appropriate theoretical formulas. Accuracy is ensured, and the time integration method, space difference method, and 
precession method are used as computer technologies to calculate in a realistic timeframe. Each of the above elements is used to construct the oil seal simulator (Amasaka, 2008; Amasaka, Tsubaki, Ymaji, \& Ueno, 2009; Leo, Annos, \& Oscarsson, 2004; Mitsuhashi, Takeoka, Tanabe, Yamaji, \& Amasaka, 2007; Nozawa, Yamashita, \& Amasaka, 2011).

\section{CAE Analysis of Oil Seal Leaks in the Drive Train}

Two-dimensional (2D) analysis. Utilizing the above-mentioned technological elements, numerical analysis was conducted through two-dimensional analysis to determine the behavior of oil around the minute projections. The space where oil is trapped between the seal and the shaft around minute projection AA' and minute projection BB' was represented and analyzed. The results are shown in Figure 8. This 2D analysis showed that shear stress was generated in the fluid oil due to shaft rotation, and backflow was occurring on the seal side via the oil path between the minute projections.

Further, when looking at ringed areas, it was found that the amount of backward oil flow from section AA' was less than the amount from section BB'. This indicates that in terms of the direction of oil flow, the sharper the angle of minute protrusions, the more backflow volume occurs.

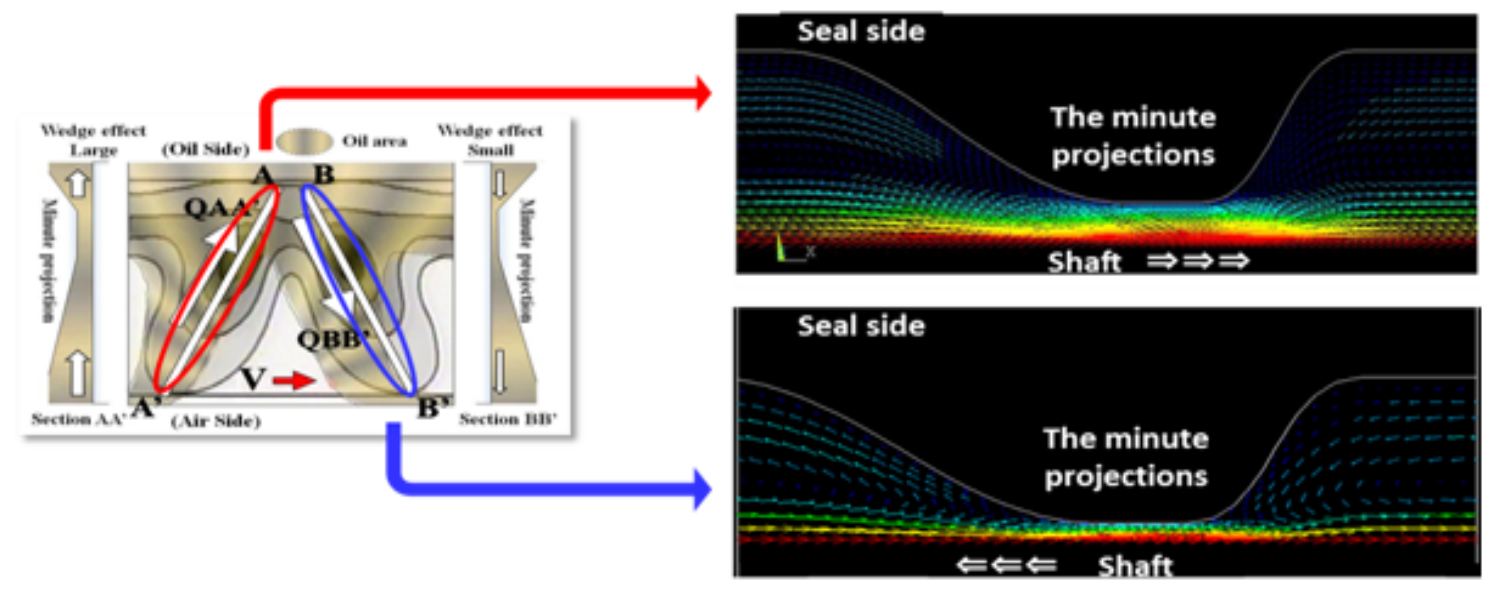

Figure 8. Example of the 2D analysis.

Three-dimensional (3D) analysis. Based on the findings from the 2D analysis, 3D analysis was conducted for the whole sliding surface model, taking into account oil flow in the third dimension (depth). Numerical simulation was conducted for the film of oil on the sliding surface with regard to the three types of seal used in the visualization experiments.

With the two parameters $A_{R}$ and $X_{G}$ incorporated into the basic model, an analysis model was constructed for each type of oil seal. The purpose of these models is to understand oil behavior and pump volume when the axis rotates. The basic and analysis models for each type of oil seal are shown in Figure 9. First, the behavior of oil under certain conditions (such as a given shaft rotational speed) was identified using the analysis model. The results are shown in Figure 10. Based on these outcomes, it can be concluded that the movement of the axis pulls oil along, and that oil flow is deflected when it hits minute protrusions.

Next, the volume of oil running across the oil side section and atmospheric side section was calculated in order to quantitatively grasp oil leak stoppage in terms of pump volume. The results of these calculations are shown in Figure 11. The analysis revealed that the amount of oil flow across individual sections could be 
computed by calculating element flow speed at the oil side and atmospheric side sections, and then multiplying this speed by the area of that element.

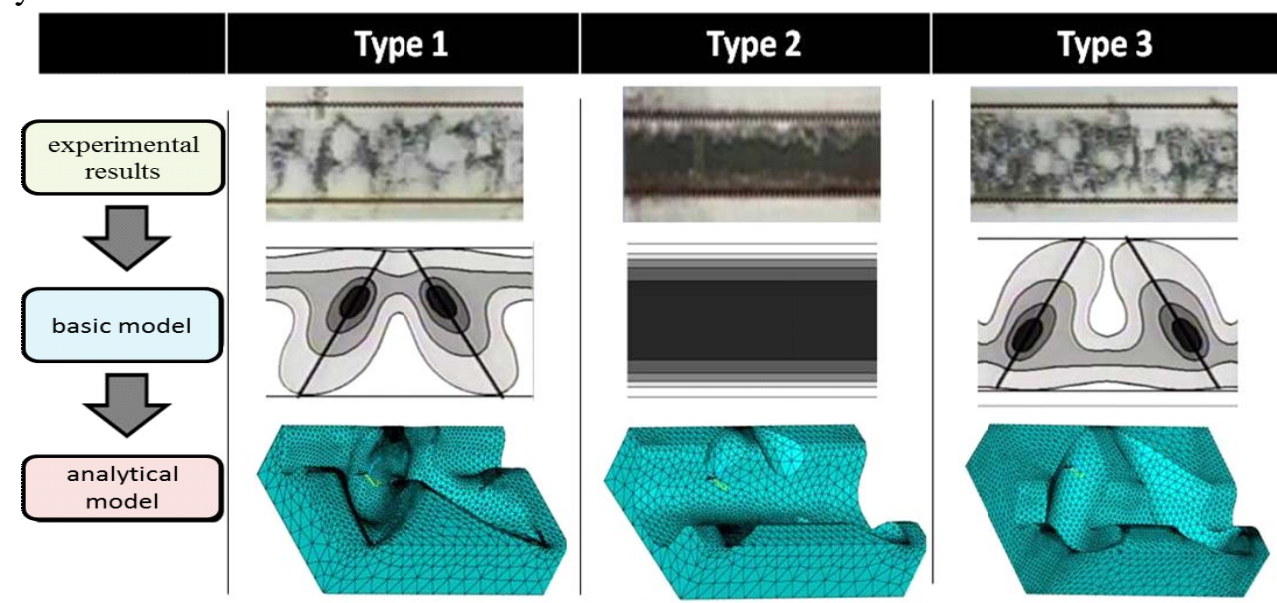

Figure 9. Basic modelten and analytical model.

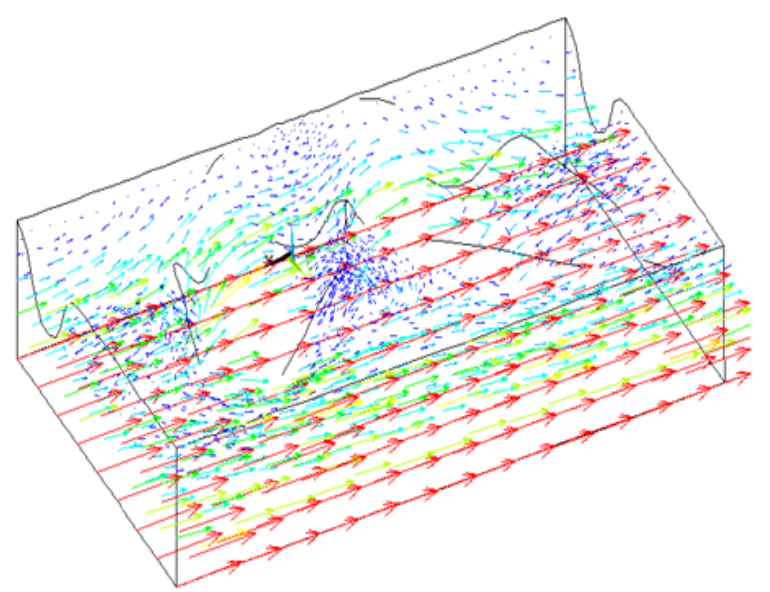

Figure 10. Example of the 3D analysis (vector representation).
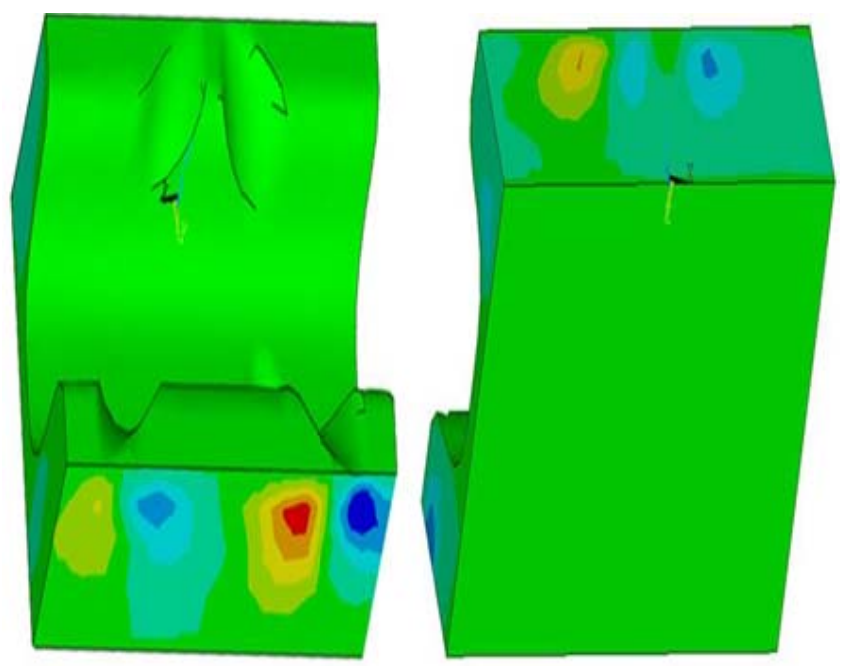

Figure 11. Example of the 3D analysis (contour figure). 
The results for each type of oil seal are shown in Table 3. The table shows positive values for elements flowing from the atmospheric side towards the oil side; in other words, oil flows towards the oil side when the calculated value is positive, and towards the atmospheric side when calculated value is negative. It is important to pay attention to the direction that the oil between the seal and the axis is flowing-meaning that it is critical to know whether the oil side surface and atmospheric side surface add up to a positive or negative total.

The results of this analysis indicate that only the first type of oil seal maintains oil volume from the atmospheric side to the oil side, while the second and third types show slightly more oil running from the oil side to the atmospheric side-thus reproducing the results of the visualization experiment.

Table 3

Results of Analysis of Oil Volume Across Sections

\begin{tabular}{llcc}
\hline & Type 1 & Type 2 & Type 3 \\
\hline Oil side section & 2.85777068 & 2.21671950 & 1.97156421 \\
Atmospheric side section & 0.683793885 & -4.24380419 & -2.26039647 \\
Total & 3.541564565 & -2.02708469 & -0.28883226 \\
\hline
\end{tabular}

Consideration. This section describes an oil flow analysis conducted using a 3D model of the three types of oil seals used in the visualization experiment. First, this allowed the researchers to understand oil behavior towards the back direction - a consideration that was not possible with a 2D analysis.

This analysis identified the flow of oil as it avoided the minute protrusions. The avoidance flow happened in line with the shape of the minute protrusions and differences were noted for each type of seal, leading to the idea that oil flow relies on protrusion form. A particularly significant difference was noted in true contract area in ratio $A_{R}$. It was concluded that $A_{R}$ impacts oil behavior, making it likely that the presence of minute surface roughness is a factor that changes the direction of oil flow from the direction of axial rotation towards the oil or atmospheric side.

In terms of the pump volume that this analysis was designed to clarify, the first type of pump was the only one that succeeded in achieving a hermetic seal-which it achieved by maintaining oil volume from the atmospheric to the oil side. This flow from the atmospheric side to the oil side is the pump volume. The second and third types of oil pump were unable to maintain pump volume, resulting in leaks. The next chapter presents a comparative analysis of the results of this analysis and actual experimental data as a means of verifying the above results. The mechanism causing oil leaks in drive train oil seals is also considered based on the insights gathered from this analysis.

\section{Verification}

Both the visualization experiment and CAE analysis ended up with similar results: The first oil seal successfully created a hermetic seal, while the second and third seals resulted in oil leaks. At the same time, an actual machine with similar criteria successfully created a hermetic seal with a pump volume of one to 20 $\mathrm{cm}^{3} /$ hour ${ }^{[1-5]}$. Aligning the units used for the values calculated in this analysis (Formula (3)) suggests that the pump volume values are valid.

$$
3.541564565 \mathrm{~mm}^{3} / \mathrm{s}=12.74963243 \mathrm{~cm}^{3} / \mathrm{hr}
$$

Another conclusion that can be reached by looking at the analysis results is that oil flow conditions on the atmospheric side are what make the difference between a type 1 seal, which successfully creates a hermetic seal, and a type 2 or 3 seal, which suffers from oil leaks (see Figure 11). The condition of oil inflow and outflow is 
thus a significant factor in the presence of leaks. One common point is that when leakage flow occurs, signs of oil being drawn in appear in the area immediately surrounding the leak.

This is likely to be one of the reasons that the balance of oil inflow and outflow shifts towards the atmospheric side. The results of a calculation of the volume of oil flow at the oil side and central section of the atmospheric side using the analysis model are shown in Table 4.

Table 4

Flow Volume Comparison: Central Section vs. Atmospheric Side Section

\begin{tabular}{lllll}
\hline & & Type 1 & Type 2 & Type 3 \\
\hline \multirow{2}{*}{ Central section } & Inflow direction & 3.123374143 & 0.42312115 & 2.032577124 \\
& Outflow direction & 2.357885132 & 1.86210332 & 2.982331467 \\
\multirow{2}{*}{ Atmospheric side section } & Inflow direction & 3.245637538 & 2.98561211 & 5.235524511 \\
& Outflow direction & 2.561843653 & 7.2294163 & 7.495920981 \\
\hline
\end{tabular}

The table compares the volume of oil flow in the inflow and outflow direction at the central section and the atmospheric side section. The results indicate approximately the same values for the center and atmospheric side sections in the first type of oil seal, and in the second and third types (those likely to develop leaks), a decreased volume of oil flow in both directions for the central portion compared to the volume of flow for the atmospheric side section.

In other words, oil inflow and outflow conditions were confirmed to be similar to those in the lower part of Figure 12. This indicates that an inflow/outflow balance that has shifted towards the atmospheric side is a key factor in generating oil leaks (Amasaka, 2010).

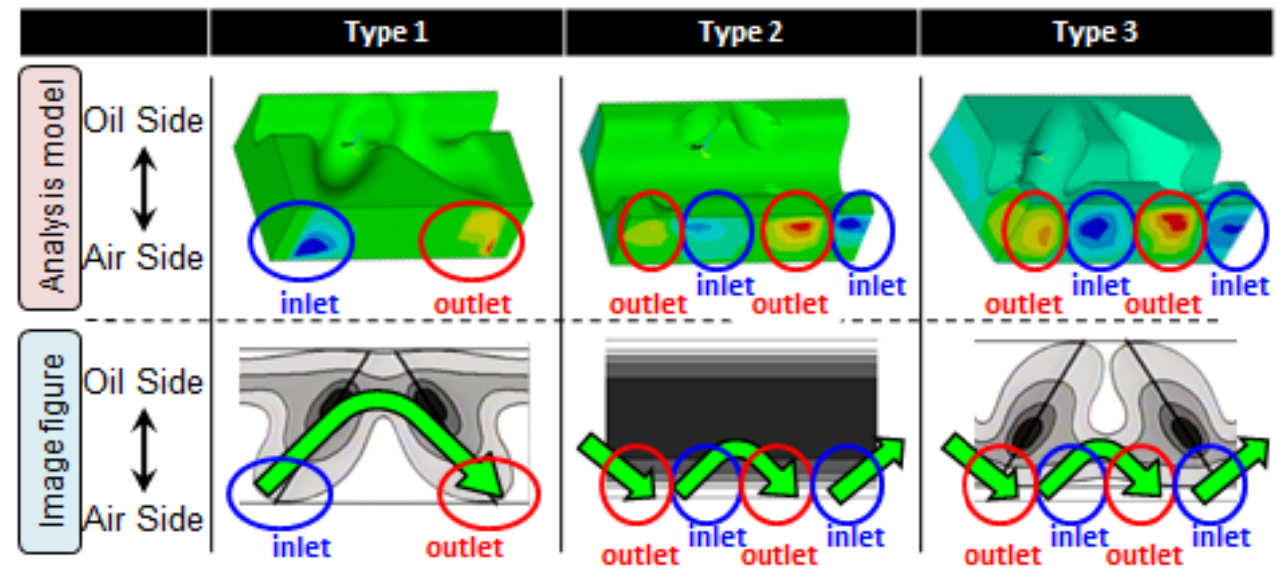

Figure 12. Comparison of the analysis result.

\section{Conclusions}

In this study, technical problems where the structure of the problem is unknown are a cause for concern in the automotive manufacturing industry. One such problem is an automotive transaxle oil seal leakage problem, which the authors solved using a "problem identification—visualization experiments—logical reasoning—CAE analysis—design” process.

In the visualization experiments, it is confirmed that there exist a space between the seal contact surface and surface touches by oil. This result was based on numerical simulation. In the preliminary step of CAE 
analysis, the utilization of CAE analysis to grasp the technical factors model was conducted and confirmed. Later, the effect of pump volume and contact point of seal was confirmed in 2D analysis to grasp the movement of oil and the seals axles. Plus, based on 3D simulation analysis, the contact area, $A_{R}$ and the degree of oil movement $X_{G}$ were described and made in three types of seals for model analysis.

As a result, based on the calculation of pump volume for different type of oil seals was resulted in stopping of leakage of oil. Plus, the results for stopping of oil leak based on the balance flow of oil and the flow direction of air is also found out.

As stated above, it is understood that the point of contact and pump volume was related and this could contribute towards seals quality design. Plus, the utilization of CAE analysis in prediction phase of realized design development is also possible.

\section{References}

Amasaka, K. (2007). Highly reliable CAE model, the key to strategic development of advanced TDS. Journal of Advanced Manufacturing Systems, 6(2), 159-176.

Amasaka, K. (2008). An integrated intelligence development design CAE model utilizing new JIT, application to automotive high reliability assurance. Journal of Advanced Manufacturing Systems, 7(2), 221-241.

Amasaka, K. (2010). Proposal and effectiveness of a high quality assurance CAE analysis model, innovation of design and development in automotive industry, current development in theory and applications of computer. Science, Engineering and Technology, 2(1/2), 23-48.

Amasaka, K., \& Osaki, S. (2003). Reliability of oil seal for transaxle-A science SQC approach in Toyota-Case studies in reliability and maintenance (pp. 571-588). New Jersey: John Wiley \& Sons, Inc..

Amasaka, K., Tsubaki, H., Yamaji, M., \& Ueno, T. (2009). Establishment of bolt tightening simulation system for automotive industry: Application of the highly reliable CAE model. International Business and Economics Research Journal, 8(5), 57-67.

Amasaka, K., Yamaji, M., \& Ito, T. (2010). Optimized design using high quality assurance CAE-Example of the automotive transaxle oil seal leakage. Proceedings of the 9th WSEAS International Conference on System Science and Simulation in Engineering (pp. 227-232). Iwate Prefectural University, Iwate, Japan.

Ito, T., Sato, M., Yamaji, M., \& Amasaka, K. (2010). An analysis of bottleneck technology by using experiments and CAE: Example of the automotive transaxle oil seal leakage. International Business \& Economics Research Journal, 9(12), 123-129.

Leo, J. D. V., Annos, N., \& Oscarsson, J. (2004). Simulation based decision support for manufacturing system life cycle management. Journal of Advanced Manufacturing Systems, 3(2), 115-128.

Magoshi, Y., Fujisawa, H., \& Sugiura, T. (2003). Simulation technology applied to vehicle development. Journal of Society of Automotive Engineers of Japan, 57(3), 95-100.

Mitsuhashi, T., Takeoka, S., Tanabe, T., Yamaji, K., \& Amasaka, K. (2007). Interim report of WG4's studies in the Japanese society for quality control research activity of simulation and SQC (the 4th report): Concept of high reliability CAE for a fixed bolt (Part 2). Proceedings from the 83rd Technical Conference (pp. 107-110), Tokyo, Japan.

Nakamura, K. (1987). Sealing mechanism of rotary shaft lip-type seals. Tribology International, 20(2), 90-101.

Nakamura, K., Kameike, M., \& Ono, S. (2000). The helical seal: Sealing concept and rib design. Sealing Technology, 77, 7-11.

Nozawa, Y., Yamashita, R., \& Amasaka, K. (2011). A CAE method of design optimization for quality assurance: Analyzing cavitation caused by metal particles in the transaxle. Proceedings of the 2011 International Symposium on System Integration (pp. 1383-1387), Kyoto University, Kyoto, Japan.

Steinberg, D. M. (1996). Robust design: Experiments for improving quality. In S. Ghosh, \& C. R. Rao (Eds.), Handbook of Statistics 13, North Holland, Amsterdam: Elsevier.

Takahashi, T., Ueno, T., Yamaji, M., \& Amasaka, K. (2010). Establishment of highly precise CAE analysis model—An example of the analysis of automotive bolts. International Business \& Economics Research Journal, 9(5), 103-113.

Toda, A., Nakamura, K., Ono, S., \& Sato, Y. (1999). A study of the sealing mechanism of radial lip seal with helical ribs measurement of the lubricant fluid behavior under sealing contact. SAE Technical Paper Series, 1999-01-0878. 